\title{
A PERSONAL COMPUTER-BASED MONITORING AND CONTROL SYSTEM FOR ELECTRON ACCELERATORS
}

\author{
L. A. Van Ausdeln, K. J. Haskell, J. L. Jones, INEEL, Idaho Falls, ID
}

\section{Abstract}

A virtual instrument (VI) has been designed to provide monitor and control function for a selectable-energy, electron accelerator at the Idaho Accelerator Center (IAC). The virtual instrument is written using the National Instruments LabVIEW[1] graphical programming language for operation on an IBMcompatible, Windows-based, personal computer. All data acquisition is accomplished using National Instruments data acquisition hardware interface (NI-DAQ). The VI monitors various electronically isolated accelerator parameters: electron source gun filament voltage, magnetron current, beam current, vacuum pressure, radiation exposure, and accelerator cavity assembly temperature. The radiation exposure, vacuum pressure, and cavity assembly temperature readings are used to control safety interlocks with user adjustable trip points. The VI provides manual controls for magnetron high voltage, electron source gun filament voltage, and RF tune, and can also deflect the electron beam across an off axis faraday cup to obtain beam energy profile information. This monitor and control system has been designed to enable customization with other electron accelerators so as to enhance operational predictability and repeatability.

\section{INTRODUCTION}

An electron accelerator[2] located at the Idaho Accelerator Center in Pocatello, Idaho, is used in support of several nondestructive evaluation (NDE) programs based at the Idaho National Engineering and Environmental Laboratory (INEEL). The predictable and highly repeatable operation of an electron accelerator is very important to the development of these NDE technologies. This type of accelerator operation is facilitated by the use of a real time PC-based virtual instrument to monitor and control key accelerator and beam parameters. Such an instrument has been developed using National Instruments (NI) LabVIEW graphical software package, NI-provided isolation interface components, and a LAB-PC-1200 NI-DAQ. Figure 1 presents the associated system components and their interconnections. Arrowheads directed into the LabVIEW interface, which includes the processor(cpu), signify monitoring functions. Arrowheads directed toward hardware signify accelerator control functions. Text in italics identify interlock control parameters and associated signals.

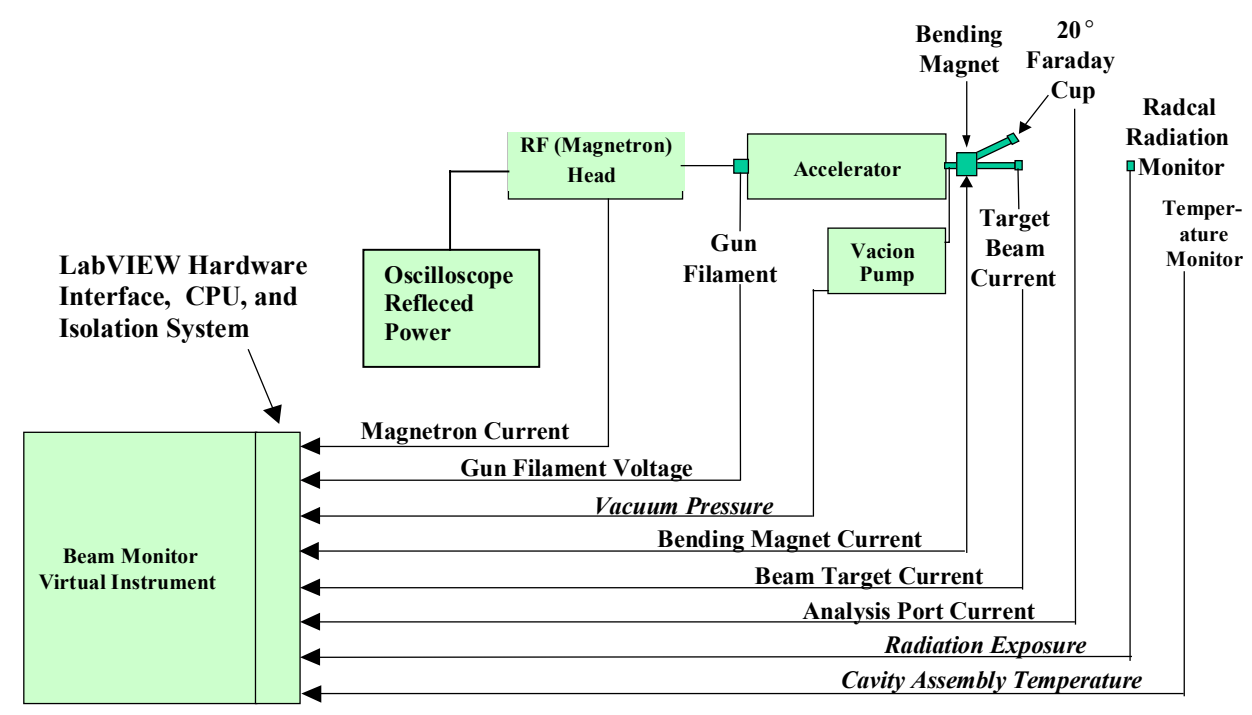

Figure 1: Beam Monitor Virtual Instrument and Accelerator Hardware 


\section{SOFTWARE}

LabVIEW V4.1 graphical program language was selected for its ability to allow the user to create a front panel user interface, or virtual instrument. Graphical programming languages allow the designer to select objects (icons) to perform required functions. LabVIEW and NI-DAQ allows hardware configuration, output display, and data acquisition to be implemented in a short time period.

\section{ISOLATION SYSTEM}

Signal isolation (Figure 2) is accomplished by incorporating NI-provided 5B08 backplane, 5BXX series analog I/O isolation modules (left side), an

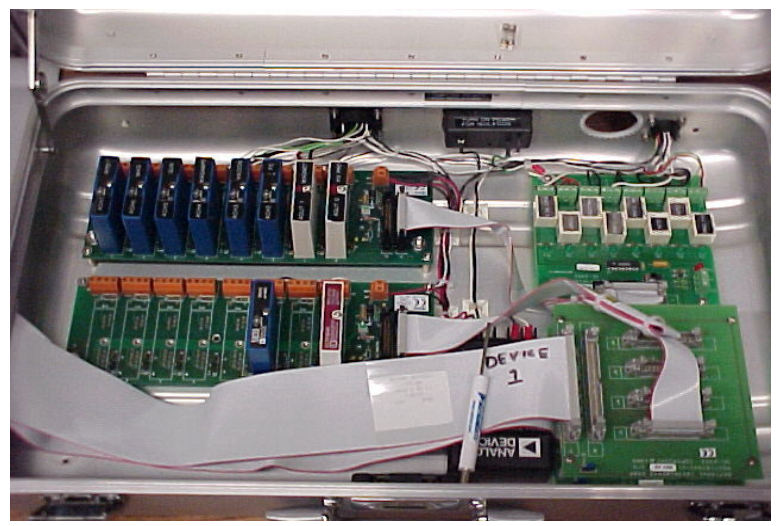

Figure 2: Isolation components and portable case.
An NI SC-2053 adapter (lower right) is used to interface isolation components to the 1200 family of NIDAQ. Use of the isolation system resulted in enhanced signal stability and reduced signal-to-noise ratios, both of which are critical to optimal performance and maximal repeatability.

\section{VIRTUAL INSTRUMENT}

The virtual instrument monitors several key accelerator parameters: magnetron current, electron source gun filament voltage, vacuum pressure, beam target current, radiation exposure, bending magnet current, and analysis port current. The latter two parameters are used to measure the electron beam energy by measuring the beam momentum, or effectively, the beam energy profile. The latter is accomplished by automatically ramping (2 Amps/s) the bending magnet current from 0 to (as much as) 18 amperes, and then to 0 again. The electron beam crosses a $20^{\circ}$ off-axis faraday cup whose current is monitored via the analysis port signal.

The virtual instrument user display is shown in Figure 3 , and consists of several sections:
A. Graph Section
B. Signal Interlock Section
C. Status/Toggle Section
D. Faraday Cup Signal Section
E. Monitored Beam Parameters Section
F. Save/Load Beam Scan Section
G. RF Tune Section

NI SC-20XX series isolated digital output device (top right), power supply and associated cabling (lower center), all mounted in a portable case.

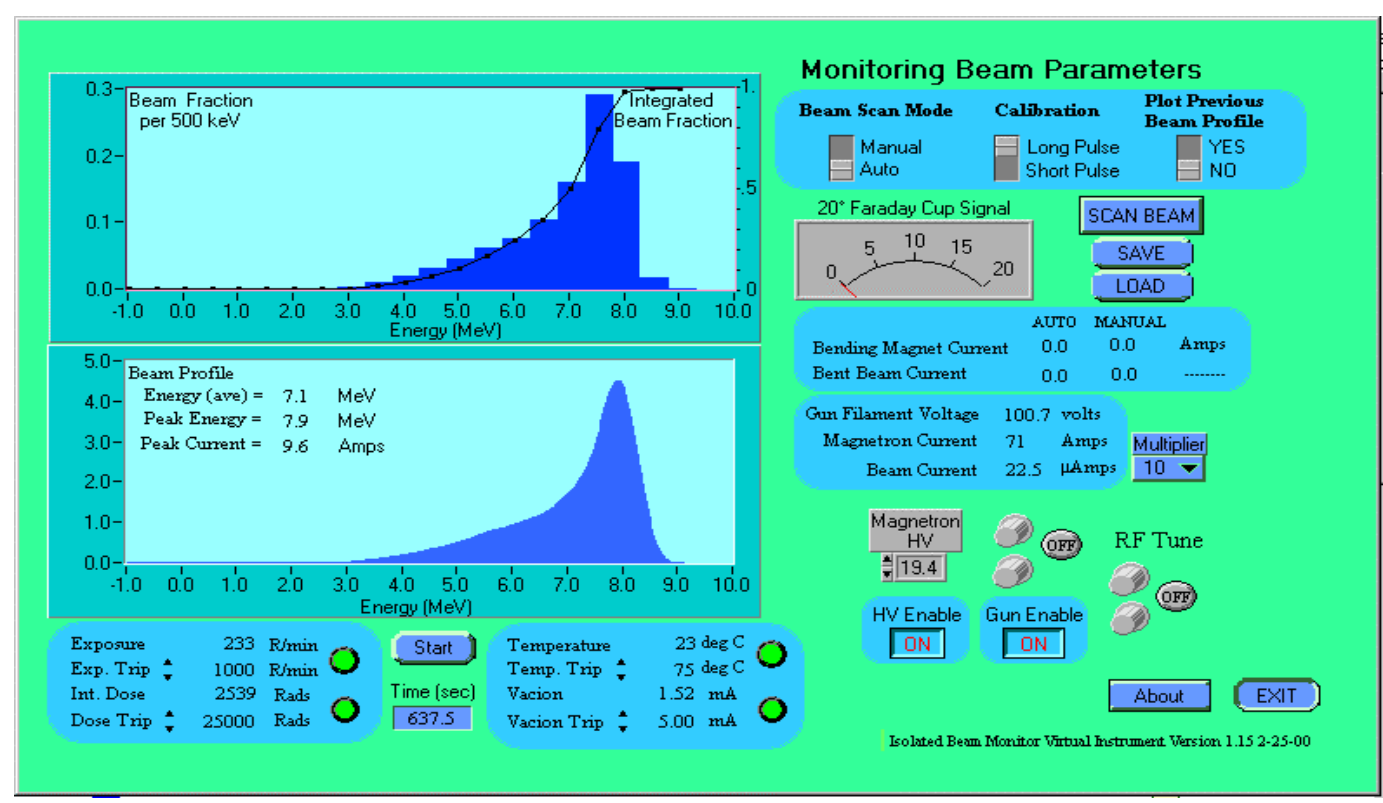

Figure 3: Virtual Instrument User Display 


\section{A. Graph Section(left half)}

This portion consists of two graphs: Beam Fraction profile (upper), and Beam Energy profile (lower). Beam Energy graph is the plot of the beam current response measured in the accelerator's $20^{\circ}$ faraday cup during an auto beam scan. The average energy is a simple average of displayed energy values. The peak energy is the value of the electron beam energy corresponding to the maximum measured beam current. The peak current is the faraday cup current reading corresponding to the peak energy. The upper graph displays the fraction of total beam flux per $500 \mathrm{keV}$ interval, as well as the integrated fraction of total beam energy below the corresponding beam energy.

\section{B. Signal Interlock Section [Lower left]}

Displayed in this section are four safety interlock control parameters. Each of the monitored values: exposure, integrated dose, accelerator temperature (temperature), and vacuum pressure (vacion), is displayed separately along with their user-adjustable trip points. When the virtual instrument is started, each of the trip point values is initialised to a default value. An indicator light, adjacent to each trip setting, will change from green to red when a trip point is exceeded. The VI automatically terminates accelerator operation when a trip point is exceeded. The integrated dose and time displays may be reset to zero using the Start button. Dose is read from a RadCal, Inc. ionisation chamber positioned forward of the accelerator.

\section{Status/Toggle Section [Upper right]}

In this section of the instrument, one of two messages are displayed indicating the current mode of the instrument: "Monitoring Beam Parameters" or "Auto Beam Scan Mode". Below this are three toggle switches to select the beam scan mode, either "Auto" or "Manual" (used for diagnostics), the beam pulse mode, either "Short Pulse" or "Long Pulse", and whether or not the previous beam scan is to be plotted when a subsequent auto beam scan is performed. It also consists of a push-button "Scan Beam" to initiate an auto beam scan.

\section{Faraday Cup Signal Section [Upper right]}

Located below the Toggle Section is a needle gauge showing the faraday cup current on the $20^{\circ}$ faraday cup during a beam scan. The corresponding bent beam data is shown in digital format below this gauge, along with the current applied to the bending magnet.

E. Monitored Beam Parameters Section [Right center]

Digital values of three important accelerator/beam parameters are displayed:

Gun Filament Voltage: Corresponds to the electron source extraction voltage

Magnetron Current: Corresponds the RF power supply average current

Beam Current: Corresponds to the target average beam current. Adjacent to the Beam Current display is a control to set the "Multiplier" to match a multiplier setting as used on an Ortec 439 digital current integrator.

\section{F. Save/Load Beam Scan Section [Upper right]}

Adjacent to the Monitored Beam Parameters Section are two buttons the user may use to either save the most recent auto beam scan, or read in and display a previously saved beam scan.

\section{G. Tune Section [Lower right]}

Immediately below the Monitored Beam Parameters Section are controls used to manually tune various beam parameters. Activation of the HV Enable button causes a control to appear that allows user control of the magnetron high voltage. Activation of the Gun Enable button causes two buttons to appear. The user may then adjust these two controls to either raise or lower the voltage to the electron source gun filament. Various gun filament control resolutions are available for use: $0.1,1.0$, and 10.0. Selecting one of these resolutions allows the user to control how much of a voltage change occurs each time a control is pushed. Activation of both the $\mathbf{H V}$ Enable and Gun Enable buttons causes two additional RF Tune control buttons to appear. These allow the user to fine-tune the RF reflected power, as viewed on an external oscilloscope, to optimise beam flux.

\section{FUTURE DEVELOPMENT}

Future development of the LabVIEW software, and expanding hardware and interface capabilities, will allow total automated control and monitoring. For instance, four energy levels of output for the accelerator are normally used: $6,8,10$, and $12 \mathrm{MeV}$. The VI would contain a "Button" for each utilized energy setting. Upon energy selection, the system will automatically enter a setup mode for that beam energy, set the safety interlocks, adjust controls to desired levels, perform beam analysis, and tune itself into operational balance. Automatic monitoring and control could remain enabled to ensure the accelerator maintains the desired beam energy.

\section{SUMMARY}

National Instruments LabVIEW and NI-DAQ hardware provides an inexpensive, fully supported, PC-based solution to accelerator monitoring and control. LabVIEW basics and the associated hardware setup were straightforward. If expansion is desired, other platforms for large-scale DAQ systems should be investigated.

\section{REFERENCES}

[1] National Instruments Corporation, "Measurement and Automation 2000," Austin, Texas.

[2] J.L. Jones, et. al., "Pulsed Photoneutron Interrogation: The GNT Demonstration System," INEEL report, WINCO1225, October 1994. 